\title{
An alternative method to study cross-flow instabilities based on high order dynamic mode decomposition
}

\author{
Cite as: Phys. Fluids 31, 094101 (2019); doi: 10.1063/1.5110697 \\ Submitted: 23 May 2019 • Accepted: 9 August 2019 • \\ Published Online: 3 September 2019
}

Soledad Le Clainche, ${ }^{1, a)}$ (D) Zhong-Hua Han, ${ }^{2}$ (D) and Esteban Ferrer ${ }^{1}$ (D)

\begin{abstract}
AFFILIATIONS
${ }^{1}$ School of Aeronautics, Universidad Politécnica de Madrid, 28040 Madrid, Spain

${ }^{2}$ School of Aeronautics, Northwestern Polytechnical University, Xian 710072, People's Republic of China
\end{abstract}

a) Author to whom correspondence should be addressed: soledad.leclainche@upm.es

\begin{abstract}
We present a novel method for the determination of $N$-factors in cross-flow transition scenarios. The method considers numerical simulations, in which a turbulent model is applied downstream from a predetermined point and solves a laminar flow upstream from this point. The solution is postprocessed using higher order dynamic mode decomposition to extract the leading spatial mode in several small sections along the streamwise direction. The spatial evolution of the amplitude of this mode will determine the $N$-factor. The results presented are compared with experimental measurements and linear stability theory, showing the good performance of this novel method, which does not assume parallel flow assumptions, is automatic and computationally efficient.
\end{abstract}

Published under license by AIP Publishing. https://doi.org/10.1063/1.5110697

\section{INTRODUCTION}

Commercial aircrafts are designed to minimize the wave drag derived from transonic effects, at cruise flow conditions. The high drag penalties related to the appearance of shocks over wings together with the sudden changes in stability and maneuverability have pushed the manufacturers to limit cruise speeds and find alternative configurations to avoid the drag increase due to transonic effects. The widely spread palliative is sweeping the wings. By doing so, the incoming Mach number (or associated velocity) at the wing leading edge is roughly decreased as $M a_{i}=M a \cos (\Lambda)$, where $M a_{i}$ and $M a$ are the incident and free stream Mach numbers, respectively, and $\Lambda$ denotes the wing sweep angle. This modified wing configuration enables higher cruise speeds and has been adopted as the mainstream design.

Large sweep angles come at the cost of an early transition from laminar to turbulent flow in the boundary layer, complicating the design of laminar wings with sweep. When the sweep angle is small, Tollmien-Schlichting (TS) waves govern the transition mechanism in boundary layers. This viscous instability was first theoretically shown by Tollmien ${ }^{45}$ and Schlichting. ${ }^{38}$ TS waves develop in boundary layers and grow exponentially in space (but are temporally stable) until nonlinear interactions dominate and lead to the turbulent regime.

For large sweep angles, the mean flow outside the boundary layer curves and promotes a secondary flow and an associated threedimensional inflection point. ${ }^{34}$ As a consequence, the primary instability is a low-frequency crossflow wave, which saturates to lead to a steady spanwise modulation (i.e., a periodic spanwise flow). On the one hand, in environments with low amplitude disturbances (e.g., low turbulent environments and in flight condition), a secondary stationary instability develops upon the shear-layer cross-flow vortices resulting from the first instability, which dominate the transition mechanism. On the other hand, if the environment presents high amplitude disturbances (e.g., high turbulence levels), then a traveling secondary instability dominates. Further description may be found in Refs. 3, 4, 40, and 41. Secondary instabilities dominate the cross-flow transition scenario over swept wings. A characteristic of the secondary instabilities is their rapid spatial growth and that their frequencies exceed the typical range of TS waves for comparable flow conditions. ${ }^{31,19}$ This type of transition scenario is called cross-flow transition. 
Arnal and Casalis, ${ }^{1}$ Bippes, ${ }^{2}$ Saric et al., ${ }^{34}$ Saric et al., ${ }^{35}$ and Reed et $a l .{ }^{33}$ provide a complete overview on the dominant flow physics arising from swept-wings. These reviews provide a summary of receptivity mechanisms by which external disturbances (e.g., free stream turbulence or roughness) or flight parameters (e.g., Mach number or Reynolds number) affect the stability and transition of three-dimensional boundary layers, and provide guidelines to its control. ${ }^{5,9,49}$

Researchers have studied cross-flow transition by means of various techniques. Local linear stability analysis and the $e^{N}$ method have been successful on predicting TS type transition, but the underlying linearity assumption has limited its ability to predict the onset of turbulent flows when dominated by nonlinear instabilities (e.g., cross-flow transition scenarios). More sophisticated techniques include two-dimensional eigenfunctions ${ }^{29}$ or Floquet analysis. ${ }^{47}$ More recently, Parabolized Stability Equations (PSE) and nonlinear PSE (NPSE), see Herbert ${ }^{16,17}$ and Tempelmann et al., have shown success for flows that respect the underlying assumptions of the models. In PSE analysis, ${ }^{17}$ the boundary layer is assumed to grow slowly in the disturbance-propagation direction and second derivatives in that direction are neglected such that parabolized stability equations can be derived, enabling us to spatially march the solution. These weakly parallel assumptions are not strictly valid for cross-flow instabilities. Additionally, these techniques typically require the choice of a particular mode or set of modes to perform the analysis.

In any case, these techniques may find difficulties in predicting mode interactions. Malik et al. ${ }^{30}$ showed that when the stationary cross-flow amplitudes are of the order of the basic state quantities, there can exist as many as seven unstable modes (i.e., type I, type II, or a combination of both secondary type instabilities). Additionally, multiple peaks in the growth-rate plot can combine into a single mode ${ }^{30}$ and can lead to the crossing of modes with other modes (i.e., modal degeneracy), see Koch. ${ }^{18,43}$ These mechanisms may limit the usability of the techniques that use modeled instabilities and require a priori knowledge of the unstable modes governing transition.

Following the spirit of minimizing modeling and underlying assumptions, we propose a novel technique to study cross-flow instabilities. The method relies on performing a base-flow simulation where the transition point is fixed. After this point, a turbulent model is utilized to avoid the necessity of resolving turbulent structures, while before the transition point a fine enough grid is used to compute a direct simulation (no modeling), only for the laminar region. The simulation of the laminar region is not computationally expensive but includes the information on small disturbances that can spatially grow and govern the transition scenario. In particular, TS and secondary instabilities can be extracted. In our work, these flow structures are analyzed using a variant of dynamic mode decomposition, ${ }^{39}$ called High Order Dynamic Mode Decomposition (HODMD), ${ }^{25}$ which performs the analysis in space and enables the extraction of perturbation amplitudes and associated wavenumbers. Note that since our method relies on the simulation of the laminar regions, we do not need to model or introduce perturbations upon a base flow, but instead we analyze the structures that are hidden in the simulation. This technique is data-driven and enables the extraction of flow structures including primary and secondary instabilities but also allows for the interaction of modes, generalizing and complementing other techniques, such as NPSE.
In $\mathrm{Wu}$ et al., ${ }^{48}$ we proposed a similar DMD based transition prediction method for two-dimensional airfoils (governed by TS waves only) that we extend here to three-dimensional configurations and cross-flow scenarios. In that work, we compared our new method to linear stability theory (LST) using the $e^{N}$ criterion and experimental data for flows over two-dimensional airfoils. The results showed good agreement and encouraged the pursuit of more challenging scenarios. In the present work, we analyze cross-flow transition and enhance the robustness for the extraction of flow structures using HODMD combined with a robust algorithm, providing accurate and reliable results. The robustness of HODMD enables us to capture the leading modes without a priori knowledge of their shape or amplitude. We compare our results with experiments ${ }^{6}$ and LST/ $e^{N}$ solutions. ${ }^{51}$ Finally, the technique provides a computationally cheap alternative that could be potentially used in industry and may be expanded to study cross-flow instabilities in a wide range of problems. For example, the technique has the potential to provide insights into the transitional scenario where surface imperfections play an important role $^{32,42}$ in polychromatic transitional scenarios ${ }^{43}$ or when combining cross-flow with surface imperfections.

The rest of the paper is organized as follows: first, we describe the methodology in Sec. II, including details on the numerical flow solver, the base flows, and the HODMD technique. Second, in Sec. III, we include a detailed summary of the parameter selection for HODMD to obtain a robust analysis of the flow structures. Finally, the main results and conclusions are presented in Secs. IV and V, respectively.

\section{BASE FLOW COMPUTATIONS}

\section{A. The idea behind the method}

The idea behind the method is that the Navier-Stokes (NS) equations act as a filter, for an initial unrealistic flow field, leading to a realistic flow field. The numerical simulation is initialized with a random flow condition, which is by definition noisy since it is contaminated by numerical errors. The numerical simulation is advanced until a steady state is reached, and during the process, the NS equations filter the initial noise and reshape the initial flow field into a realistic flow field. When this steady state is reached, the solution is frozen in time. The instabilities that were evolving, are frozen at this point and can be analyzed using our spatial HODMD analysis. Consequently, it is not relevant if the instabilities are steady or traveling waves, since we will be studying a fixed photo of their time-evolution.

In our simulations, the existence of steady states is ensured since we simulate environments with low amplitude of disturbances (e.g., low turbulent environment), which leads to time decaying TS waves in two-dimensional airfoils and to steady secondary instabilities in three-dimensional swept wings. ${ }^{40,41}$

From the explanation above, it becomes apparent that there is no need for a receptivity mechanism ${ }^{33,35,40,41}$ to trigger or sustain instabilities, since these are already in the initial solution (noisy initial flow polluted with numerical errors) and are filtered and reshaped by the NS equations in the vicinity of the geometry (i.e., within the boundary layer). We should nonetheless point out that if the instabilities had to be triggered from an ideal base flow (free of 
errors) or sustained as time progresses, then a receptivity mechanism would be necessary.

\section{B. Numerical flow solver}

An in-house numerical solver PMNS3D is used as a flow solver in this work. PMNS3D solves the three-dimensional ReynoldsAveraged Navier-Stokes (RANS) equations for three-dimensional compressible aerodynamic problems, ${ }^{14,50}$ toward efficient numerical analysis and aerodynamic shape optimization of wings. ${ }^{13,15,51}$ The RANS equations are discretized spatially using the JamesonSchmidt-Turkel scheme with artificial dissipation and an LU-SGS scheme for time integration with the multigrid technique to accelerate the iterative convergence to steady state. The turbulence viscous coefficient is calculated by the Spalart-Allmaras one-equation turbulence model. To improve the efficiency and robustness of the compressible numerical solver for low-speed flows, a low-speed preconditioning method is included. In all cases, the formal spatial accuracy of the solver is second order. Numerical simulations are converged until the steady state is reached, which is defined when the maximum relative averaged-density residual is below $10^{-6}$. In previous work, ${ }^{48}$ we addressed the effect of the residual, in the dominant spanwise mode, to find that the influence is minimal once the residual is below $10^{-5}$. These steady states exist only because we fix the transition line at $x / c=0.6$, which is located after the "real" transition point. From the fixed point at $x / c=0.6$, the turbulence model is switch on such that the model damps transitional oscillations developed in the well resolved laminar region. Before $x / c=0.6$, laminar flow is allowed to develop freely. Therefore, TS and cross-flow instabilities can develop in space. The evolution of these instabilities in time is frozen once the steady state is reached (i.e., once the residual is below $10^{-6}$ ). Therefore, once the solution converges, we obtain a steady base flow, with frozen instabilities, with a spatial structure that can be analyzed to extract transition locations based on $N$-factors. Note that the use of $\mathrm{N}$-factors (as opposed to direct amplitudes) is here advantageous to avoid the dependence of growth rates to initial flow conditions and noise levels in the simulations. Finally, to extract the wave information (i.e., spatial growth), we used HODMD as detailed in Sec. III. Let us note that we have checked that when moving the fixed transition point from $x / c=0.6$ to $x / c=0.7$, the results are almost unchanged in that the transition location moves by less than $0.01 x / c$.

\section{Mesh and base flow for a two-dimensional low-speed laminar NLF0416 airfoil}

We first study a two-dimensional low-speed laminar NLF0416 airfoil. The base flow is obtained by solving Navier-Stokes equations with a prescribed transition $(x / c=0.6)$ at a free-stream Mach number of $\mathrm{Ma}=0.1$, Reynolds number of $\mathrm{Re}=4 \times 10^{6}$ (based on the airfoil chord), and Angle of Attack $A o A=1^{\circ}$. The C-mesh for the two-dimensional case is comparable to slicing three-dimensional meshes used later for cross-flow computations, see Fig. 1. The first grid point from the airfoil surface is located such that $y^{+}<1$ in the turbulent flow region $\left(x / c>0.6\right.$ in this case), where $y^{+}=y u_{\tau} / v$ with $u_{\tau}$ being the friction velocity, $y$ being the distance to the wall, and $v$ being the kinematic viscosity. Typical grids have 640 points in the streamline direction (512 points on the airfoils, 256 on suction and pressure sides) and 240 points in the direction normal to the airfoil surface.

This high resolution grid ensures that the streamwise flow direction is accurately resolved such as to capture TS type instabilities, which govern the two-dimensional airfoil case. An estimate for the smallest unstable wavelength of disturbances can be precomputed using Blasius solution for laminar flat plates, as detailed in the monograph by Schlichting and Gersten, ${ }^{36}$ chap. 15, p. 437 in the 8th revised and enlarged edition. First, we calculate the boundary layer thickness as $\delta=5(v x / U)^{1 / 2} \approx 0.002$ at $x=0.6 \mathrm{~m}$, for $M a=U / 340=0.1$ and $R e=U c / v=4 \times 10^{6}$, with the chord $c=1 \mathrm{~m}$. Second, we relate the smallest unstable wavelength of disturbances $\ell_{T S}$ to the boundary layer thickness: $\ell_{T S} / \delta \approx 6$, to obtain an estimate for the unstable TS wavelengths $\ell_{T S} \approx 0.01 \mathrm{~m}$ [with associated angular wavenumber $\lambda_{T S}$ $=2 \pi / \ell_{T S} \approx 628$ and dimensional wavenumber $\left.\lambda_{T S} /(2 \pi) / \approx 100 \mathrm{~m}^{-1}\right]$. The grid resolution along the streamwise direction has a spacing of 0.004 , which is fine enough to capture unstable TS waves (based on the Nyquist criterion and the estimate $\lambda_{T S} \approx 0.01$ ). To generate three-dimensional meshes, we also follow this approach and make sure that the mesh is fine enough to resolve TS waves in the laminar region.

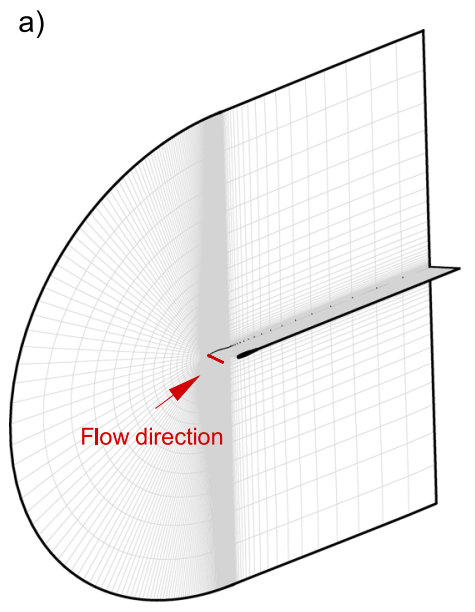

b)
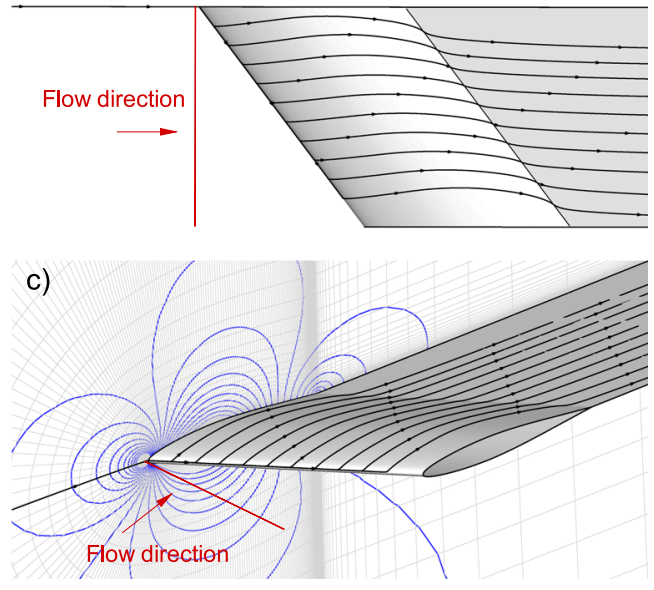

FIG. 1. (a) Computational domain for the steady base flow with sweep angle $\Lambda=40^{\circ}$, (b) top view wing streamlines on the wing, and (c) near airflow mesh and contour of static pressure (blue lines). 


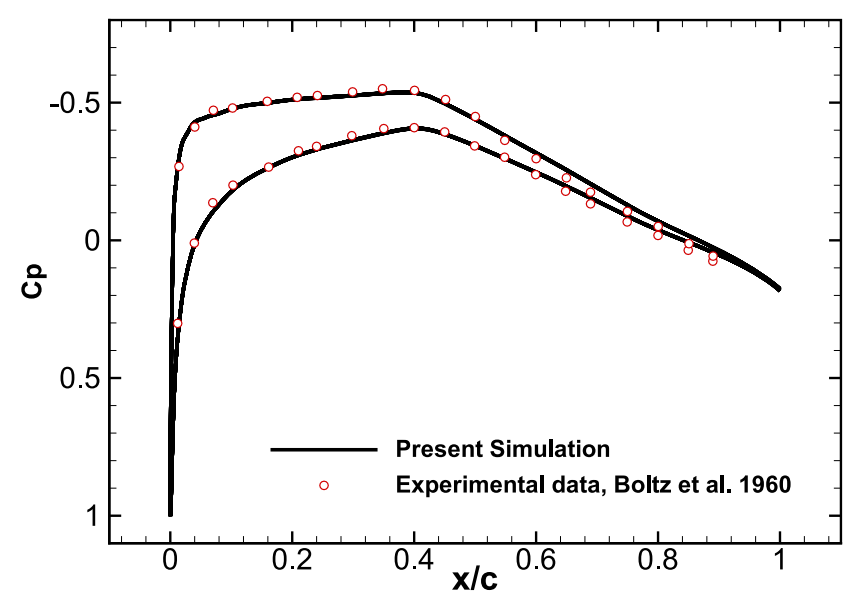

FIG. 2. Pressure coefficient distribution on a wing section for sweep angle $\Lambda=40^{\circ}$ and angle of attack $A \circ A=-1^{\circ}$ for $\operatorname{Re}=7.00 \times 10^{6}$ and $M a=0.27$.

\section{Mesh and base flow for a three-dimensional wing with sweep angle}

We extrude a laminar NACA64 ${ }_{2}$ A015 airfoil with two sweep angles $\Lambda=10^{\circ}$ and $40^{\circ}$ to generate three-dimensional wings, and embed the wing into a structured C-mesh to compute cross flow simulations. The mesh has $352 \times 104 \times 24$ elements (a total of 878.592). To discretize the spanwise direction $1 \mathrm{~m}$ long, we use 24 nodes (and periodic boundary conditions on the domain sides), providing a mesh spacing $\Delta z=1 \mathrm{~m} / 23=0.0435 \mathrm{~m}$, which is large enough to discretize the long wavelength spanwise oscillation characteristics of sweep wings with cross-flow. A mesh is shown in Fig. 1 for sweep angle $40^{\circ}$, together with the streamlines on the wing and pressure contours on a plane parallel to the free stream velocity. Additionally, the pressure coefficient is depicted in Fig. 2 for a wing section with sweep angle $\Lambda=40^{\circ}$, angle of attack $A o A=-1^{\circ}$, $\operatorname{Re}=7.00 \times 10^{6}$, and $\mathrm{Ma}=0.27$. Using the previous expression for the TS wavelength and recalculating with the new values of $\mathrm{Ma}$ and $\mathrm{Re}$, we now obtain a comparable value $\lambda_{T S} \approx 0.009$. The favorable pressure gradient characteristic of laminar airfoils (for $x / c<0.4$ ) is shown in Fig. 2 and is responsible for delaying TS transition beyond $x / c \sim 0.4$.
To compute the base flow, we fix the transition point at $x / c=0.6$, from which a turbulent model is enabled. This strategy enables the resolution of the laminar region without the need to resolve turbulent structures, which are modeled by the turbulence model. Additionally, transitional flow structures generated in the laminar region are damped by the high turbulent effective viscosity provided by the model. Note that if turbulence develops before the fixed transition point, the simulation does not converge to steady state. In these cases, we move the transition point to an earlier $x / c$. This strategy has proved useful to converge transitional flows to steady state that can be used for DMD analysis, see also Ref. 48 .

An example of the base flow obtained for a sweep angle $\Lambda=40^{\circ}$ is shown in Fig. 3, where a periodic spanwise flow is shown in Fig. 3(a). Using HODMD, we will seek secondary perturbation upon this spanwise periodic flow.

Finally, various flow conditions are considered and summarized in Table I. For these conditions, transition point locations will be obtained using the HODMD method and compared to experimental values in Sec. IV.

\section{MODE EXTRACTION USING HODMD}

\section{A. Higher order dynamic mode decomposition algorithm}

Higher order dynamic mode decomposition (HODMD) $)^{25}$ is an extension of classical dynamic mode decomposition (DMD) ${ }^{39}$ that is suitable for the extraction of temporal or spatial patterns ${ }^{10,22}$ in complex flows, i.e., transient flows, ${ }^{11,12,21,27}$ and in the analysis of noisy experimental data. ${ }^{24}$ Calculations of primary and secondary flow instabilities are based on the evolution of small perturbations, several orders of magnitude smaller than the mean flow, and the use of robust and accurate tools for mode extraction proves beneficial.

To simplify the description of the method, we detail here the HODMD algorithm in the more familiar time domain version (sequence of time snapshots) to then particularize the method to spatial data sequences as used in this paper to extract spatial evolution of waves.

The main goal of the original HODMD is to decompose spatiotemporal data $\boldsymbol{v}(x, y, z, t)$ as an expansion of DMD modes $\boldsymbol{u}_{m}(x$, $y, z)$, weighted by amplitudes $a_{m}$, that grow/decay in time with rate $v_{m}$ and that oscillate in time with frequency $\omega_{m}$,

a)

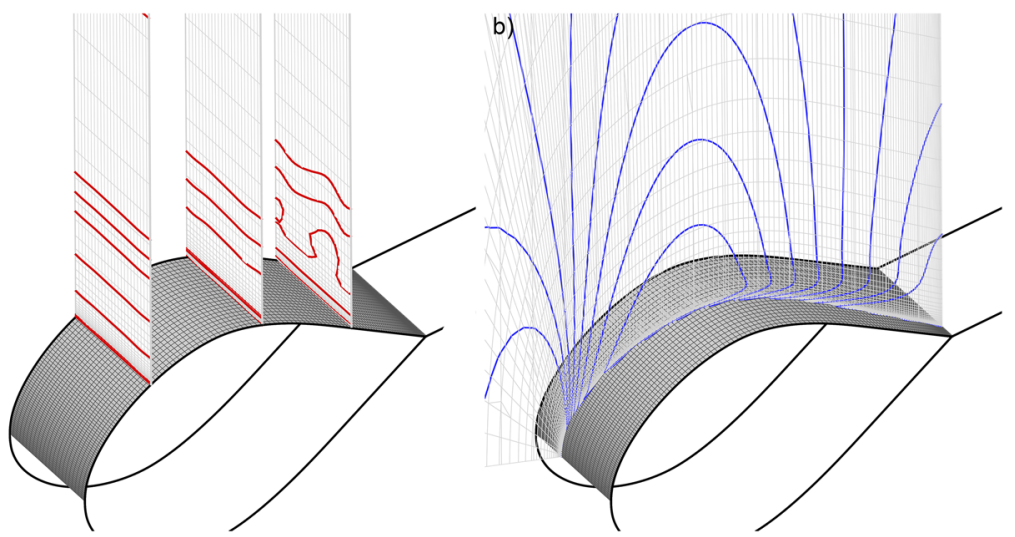

FIG. 3. Base flow for sweep angle $\Lambda=40^{\circ}$ : W-velocity contours for (a) planes parallel to the leading edge and (b) planes following the free stream velocity direction. 
TABLE I. Cross-flow wing conditions, Angle of Attack $(A \circ A)$, sweep angle $(\Lambda)$, Reynolds number $(R e)$, and Mach number $(M a)$.

\begin{tabular}{lccc}
\hline \hline AoA $(\mathrm{deg})$ & $\Lambda(\mathrm{deg})$ & $R e$ & $\mathrm{Ma}$ \\
\hline-1 & 10 & $7.00 \times 10^{6}$ & 0.27 \\
-1 & 40 & $7.00 \times 10^{6}$ & 0.27 \\
-1 & 40 & $6.35 \times 10^{6}$ & 0.27 \\
\hline \hline
\end{tabular}

$$
\boldsymbol{v}(x, y, z, t) \simeq \sum_{m=1}^{M} a_{m}^{t} \boldsymbol{u}_{m}(x, y, z) \mathrm{e}^{\left(v_{m}+i \omega_{m}\right) t} .
$$

The basic idea behind the HODMD algorithm is very simple and explained in detail by Le Clainche and Vega. ${ }^{25}$ Only some general considerations are included here.

Considering a data set of $K$ equispaced snapshots $\boldsymbol{v}_{k}=\boldsymbol{v}\left(t_{k}\right)$ (collected at time instant $t_{k}$ ), it is possible to form the following snapshot matrix:

$$
\boldsymbol{V}_{1}^{K}=\left[\boldsymbol{v}_{1}, \ldots, \boldsymbol{v}_{k}\right] .
$$

HODMD considers two main steps:

Step 1: Dimension reduction. The spatial dimension $J$ (i.e., number of grid points in the computational domain) of the original data set of snapshots is reduced to a set of linearly independent vectors of dimension $N$ using a singular value decomposition (SVD) ${ }^{37}$ that is applied to the snapshot matrix

$$
V_{1}^{K} \simeq W \Sigma T^{\top},
$$

where $\boldsymbol{W}^{T} \boldsymbol{W}=\boldsymbol{T}^{T} \boldsymbol{T}$ and $\boldsymbol{\Sigma}$ is the $N \times N$ unit matrix and the diagonal of matrix $\Sigma$ contains the singular values $\sigma_{1}$, $\ldots, \sigma_{K}$. The number of retained SVD modes, $N$, is calculated through the standard SVD-error estimated for a certain tolerance $\varepsilon$ (set by the user) as

$$
\sigma_{N+1} / \sigma_{1} \geq \varepsilon_{1} .
$$

The resulting reduced snapshot matrix of dimension $N \times K$ is written as

$$
\hat{\boldsymbol{V}}_{1}^{K}=\boldsymbol{\Sigma} \boldsymbol{T}^{\top}, \quad \text { with } \quad \boldsymbol{V}_{1}^{K}=\boldsymbol{W} \hat{\boldsymbol{V}}_{1}^{K} .
$$

Note that HODMD may be applied to temporal or spatial sequences of snapshots. While the former provides temporal information (frequency and growth/decay rates in time), the latter provides spatial information (wavenumbers and growth/decay rates in space). In this work, we retain the spatial approach, see Secs. III B and III C and particularly Eqs. (9) and (10).

When the data collected are noisy, as in the case of an experiment, ${ }^{24}$ or are represented by nonpermanent dynamics (i.e., transient modes in the transient of a numerical simulation $^{26}$ ) or a large number of small amplitude flow structures with small spatial scales, as in the case of transition to turbulent flows, ${ }^{27}$ it is necessary to increase the accuracy in the calculations. In those cases, a higher order singular value decomposition (HOSVD) ${ }^{7,20,46}$ is used instead of SVD at this step. ${ }^{27}$ The HOSVD algorithm performs a SVD in each one of the spatial directions. Thus, in this way, it is possible to better clean the data from noise or to remove not permanent modes (with negative decay rates $\delta_{m} \gg 0$ ), which can be considered as spurious modes (snapshot reduction). In this article, we have used HOSVD in order to increase the accuracy in the calculations of flow instabilities, which are based on the evolution of a small perturbation whose order of magnitude is much smaller than the mean flow.

Step 2: The DMD-d algorithm. The following higher order Koopman assumption (written in matrix form):

$$
\boldsymbol{V}_{d+1}^{K} \simeq \boldsymbol{R}_{1} \boldsymbol{V}_{1}^{K-d}+\boldsymbol{R}_{2} \boldsymbol{V}_{2}^{K-(d-1)}+\cdots+\boldsymbol{R}_{d} \boldsymbol{V}_{d}^{K-1}
$$

is applied to the reduced snapshot matrix. This technique mimics a sliding window process, which enforces the same dynamics over each one of the included submatrices, and smooths noisy solutions. Premultiplying (6) by $\boldsymbol{W}^{\top}$, we obtain a reduced Koopman operator $\hat{\boldsymbol{R}}_{k}=\boldsymbol{W}^{T} \boldsymbol{R}_{k} \boldsymbol{W}$. Using (5), Eq. (6) can be rewritten as

$$
\hat{\boldsymbol{V}}_{d+1}^{K} \simeq \hat{\boldsymbol{R}}_{1} \hat{\boldsymbol{V}}_{1}^{K-d}+\hat{\boldsymbol{R}}_{2} \hat{\boldsymbol{V}}_{2}^{K-d+1}+\cdots+\hat{\boldsymbol{R}}_{d} \hat{\boldsymbol{V}}_{d}^{K-1} .
$$

The main system dynamics (frequencies in time or wavenumbers in space, growth/damping rates, and structural shapes of DMD modes) are contained in these linear operators that can be included in a modified Koopman matrix of dimension $\mathrm{Nd} \times \mathrm{Nd}$, defined as

$$
\tilde{\boldsymbol{R}} \equiv\left[\begin{array}{cccccc}
\mathbf{0} & \boldsymbol{I} & \mathbf{0} & \ldots & \mathbf{0} & \mathbf{0} \\
\mathbf{0} & \mathbf{0} & \boldsymbol{I} & \ldots & \mathbf{0} & \mathbf{0} \\
\ldots & \ldots & \ldots & \ldots & \ldots & \ldots \\
\mathbf{0} & \mathbf{0} & \mathbf{0} & \ldots & \boldsymbol{I} & \mathbf{0} \\
\hat{\boldsymbol{R}}_{1} & \hat{\boldsymbol{R}}_{2} & \hat{\boldsymbol{R}}_{3} & \ldots & \hat{\boldsymbol{R}}_{d-1} & \hat{\boldsymbol{R}}_{d}
\end{array}\right]
$$

After some calculations [mainly based on the reduced dimension of (8)], the eigenvalues and eigenvectors of (8) are used to approximate the DMD modes, wavenumbers, and growth rates of expansion (1). Finally, the mode amplitudes $a_{m}$ are calculated by least squares fitting, and a second tolerance $\varepsilon$ is set to retain the $M$ most relevant DMD modes, exhibiting the largest amplitudes $a_{m}$. This parameter will determine the spectral complexity of the DMD expansion (1), calculated for a specific accuracy $\varepsilon$.

The benefit of HODMD lies in the resolution of the eigenvalue problem for the modified Koopman matrix $\tilde{\boldsymbol{R}}$, which enforces the dynamics contained in the delayed snapshot matrix toward the same solution. So, either the noise or not permanent modes are naturally removed from the solution, and only the permanent dynamics (i.e., flow instabilities based on the evolution of small perturbations) are maintained. It is remarkable that if $d=1$ [in Eq. (6)], the algorithm is similar to the classic DMD algorithm. The parameter $d$ identified in (6) is set by the user after some calibration, looking for robustness of the results. Hence, the method is applied several times using various parameters of $d$, $\varepsilon$, and $\varepsilon_{1}$. The calibration process is satisfied when the parameters selected provide unchanged results, in other words, when the frequency/wavenumber and damping rates calculated are similar in all the test cases carried out. ${ }^{2}$ 


\section{B. HODMD for transition predictions}

The prediction of the transition point due to cross-flow instabilities is calculated using HODMD. The method is applied to steady state solution of the three-dimensional nonlinear Navier-Stokes equations. The HODMD is applied in two steps:

- Calculations of spanwise modes. By definition, the solution of the numerical simulations is periodic in the spanwise $z$-direction. Hence, it is possible to apply HODMD in the spanwise direction to calculate spanwise harmonic modes. At this step, the three-dimensional spatial data will be decomposed following the modal expansion,

$$
v(x, y, z) \simeq \sum_{m=1}^{M} a_{m}^{z} u_{m}(x, y) \mathrm{e}^{\left(\delta_{m}+i \beta_{m}\right) z},
$$

where $u_{m}(x, y), a_{m}^{z}, \beta_{m}$, and $\delta_{m}$ are the DMD modes, related amplitudes, wavenumbers, and spatial growth rates, respectively. For spatially periodic spanwise flows (no growth or decay), we expect $\delta_{m}=0$. This is indeed confirmed a posteriori by checking the growth rates of spanwise modes, which are typically very small $\left(<10^{-4}\right)$. Note that since we consider only steady state cases, the expansion does not include time variations.

- Secondary instability in the streamwise direction and crossflow transition. To study secondary streamwise instabilities upon the spanwise periodic flow, the HODMD analysis is performed upon the spanwise DMD modes previously obtained, leading to the following expression:

$$
u_{m}(x, y) \simeq \sum_{l=1}^{L} a_{m l}^{x} \hat{u}_{m l}(y) \mathrm{e}^{\left(\gamma_{m l}+i \lambda_{m l}\right) x} .
$$

This analysis is performed sequentially, varying small sections along the streamwise direction. The main goal is to study the spatial growth of the leading modes along the various sections. The robustness of HODMD methodology ${ }^{27}$ is exploited to calculate the disturbance wavenumbers and associated amplitudes. ${ }^{25}$ It is possible to formulate the spatial growth of the leading mode as a function of the amplitudes related to each wavenumber, see below for details.

Finally, combining the two steps Eqs. (9) and (10), one may formulate the general form for the DMD expansion of the flow field as

$$
\begin{aligned}
v(x, y, z) & \simeq \sum_{m=1}^{M} a_{m}^{z}\left[\sum_{l=1}^{L} a_{m l}^{x} \hat{u}_{m l}(y) \mathrm{e}^{\left(\gamma_{m l}+i \lambda_{m l}\right) x}\right] \mathrm{e}^{\left(\delta_{m}+i \beta_{m}\right) z} \\
& \simeq \sum_{m=1}^{M} \sum_{l=1}^{L} a_{m}^{z} a_{m l}^{x} \hat{u}_{m l}(y) \mathrm{e}^{\left(\gamma_{m l}+i \lambda_{m l}\right) x} \mathrm{e}^{\left(\delta_{m}+i \beta_{m}\right) z} .
\end{aligned}
$$

To understand the relationship between spatial modal amplitudes and DMD amplitudes, we consider a small enough spatial window $(\delta x \times \delta y)$, where we compute the DMD analysis. In this window, all distinct modes ranked by a wavenumber show a distinct dominant growth rate and wavenumber such that $\left(\left.\gamma_{m l}\right|^{x},\left.\lambda_{m l}\right|^{x}\right)$ $=\left(\left.\gamma_{m l}\right|^{x+\delta x},\left.\lambda_{m l}\right|^{x+\delta x}\right)$ and identical mode shape with respect to the $y$-direction: $\left.\hat{u}_{m l}(y)\right|^{x}=\left.\hat{u}_{m l}(y)\right|^{x+\delta x}$. This is the weakly parallel flow applied to a small DMD window, and is only assumed for the small sections $(\delta x \times \delta y)$ and only during the postprocessing stage to calculate $\mathrm{N}$-factors. In our method, the weakly parallel flow is not a hypothesis of the method and it is not assumed in the simulation. Then, one may consider the definition of the $N$-factor for a section $\delta x$ as the ratio of spatial amplifications of a particular mode,

$$
N^{\delta x}=\ln \left(\frac{A(x+\delta x)}{A(x)}\right) .
$$

Additionally, we consider the definition of the spatial amplification factor $A(x)$ of eigenfunctions ${ }^{8}$ at position $x$,

$$
A(x)=\sqrt{\int_{0}^{\delta y}\left(\left(\delta u^{1^{\dagger}}\right)\left(\delta u^{1}\right)+\left(\delta u^{2 \dagger}\right)\left(\delta u^{2}\right)\right) d y}
$$

where $\delta u^{1}$ and $\delta u^{2}$ are the velocity components of two-dimensional perturbations at position $x$ and $\dagger$ denotes complex conjugation. Selecting one mode at a time from the DMD velocity decomposition (10) and introducing the expression into (13), we obtain a definition of the amplitude in terms of the DMD expansion,

$$
\begin{aligned}
A(x) & =\sqrt{\int_{0}^{\delta y}\left(u_{m}^{1}(x, y)^{\dagger} u_{m}^{1}(x, y)+u_{m}^{2}(x, y)^{\dagger} u_{m}^{2}(x, y)\right) d y} \\
& =\left|a_{m l}^{x} \mathrm{e}^{\left(\gamma_{m l}+i \lambda_{m l}\right) x}\right| \sqrt{\int_{0}^{\delta y} \hat{u}_{m l}(y) d y},
\end{aligned}
$$

where $|\cdot|$ defines the modulus of the complex number. Recalling that the DMD amplitudes $a_{m l}^{x}$ are purely real, we can simplify Eq. (15) to

$$
A(x)=a_{m l}^{x}\left|\mathrm{e}^{\left(\gamma_{m l}+i \lambda_{m l}\right) x}\right| \sqrt{\int_{0}^{\delta y} \hat{u}_{m l}(y) d y} .
$$

We can now combine (12) with (16) to define the $N$-factor as a function of the DMD amplitudes,

$$
\begin{aligned}
N^{\delta x} & =\ln \left(\frac{A(x+\delta x)}{A(x)}\right)=\ln \left(\frac{a_{m l}^{x}\left|\mathrm{e}^{\left(\gamma_{m l}+i \lambda_{m l}\right)(x+\delta x)}\right| \sqrt{\int_{0}^{\delta y} \hat{u}_{m l}(y) d y}}{a_{m l}^{x}\left|\mathrm{e}^{\left(\gamma_{m l}+i \lambda_{m l}\right) x}\right| \sqrt{\int_{0}^{\delta y} \hat{u}_{m l}(y) d y}}\right) \\
& =\ln \left(\frac{a_{m l}^{x+\delta x}}{a_{m l}^{x}}\right),
\end{aligned}
$$

where we have assumed that $\delta x$ is small and consequently $\mathrm{e}^{\left(\gamma_{m l}+i \lambda_{m l}\right)(\delta x)} \approx 1$. For a complete wing section, we can recover the overall $N$-factor $N=\sum_{l=1}^{k} N^{\delta x}$, where $k$ is the number of subdivisions $\left(A_{k}\right)$ in which to perform the DMD analyses, by applying (17) recursively for as many i-sections as necessary,

$$
N=\ln \left(\frac{A(x+\delta x)}{A(x=0)}\right)=\ln \left(\frac{a_{m l i}^{x+\delta x}}{a_{m l i}^{x=0}}\right),
$$

where we have used the properties of logarithmic functions.

Finally, we retain the following definition for the $N$-factor in terms of the DMD amplitudes: $N=\ln \left(a_{m l}^{x} / a_{0}\right)$, where $a_{0}$ is the initial reference amplitude of the perturbation (i.e., the residual level in the numerical simulations, as it is further explained below).

\section{Calibration for mode extraction using DMD}

One of the main goals of this article is to present a robust methodology that can be used as a reference, when using HODMD, to predict flow transition in a wide range of cases. To this end, it is 
necessary to first validate the method and second to present a set of parameters independent of the flow case.

Numerical simulations for the two-dimensional NLF0416 airfoil are converged to steady state (i.e., residual below $5 \cdot 10^{-7}$ ). Due to the evolution of small fluctuations, several orders of magnitude smaller than the mean flow, flow transition takes place in the streamwise direction of the airfoil, so the spatial HODMD will be applied to a set of data, equispaced along this direction. The mesh used for the numerical simulations is structured, but not equispaced, so it is necessary to interpolate the flow solution to an equispaced grid. The analysis will be performed separately in the upper and lower surfaces of the airfoil, which are both curved. To minimize interpolation errors, we interpolate the data into a grid equispaced in both streamwise and normal directions, whose separation interval needs to be sufficiently small. The value of these intervals for the streamwise and normal direction is $\Delta x=4.002 \cdot 10^{-4}$ and $\Delta y=1.2508$. $10^{-4}$, respectively. Figure 4 shows the contours of the streamwise velocity in the interpolated mesh. Figure 4 (left) shows the domain interpolated around the airfoil, while the two figures on the right show the domains for the HODMD analysis in the upper and lower surfaces. As seen, in both cases, HODMD is performed in a rectangular computational domain. The number of grid points used are 2000 for the streamwise direction $(0 \leq x / c \leq 0.8)$ and 480 for the normal direction $(0 \leq y / c \leq \pm 0.06$, the surface of the airfoil is defined for $y / c=0$ in both, the upper and lower surfaces). As shown in Fig. 4, it is possible to represent the curved domain as a simplified planar domain assuming a small error, since the equispaced interval set in both directions is small.

Once the equispaced mesh has been constructed, we can calibrate the method. This is performed in three steps:

- Selection of the region for the HODMD analysis. Both, the normal and streamwise components, play a role in the selection of the area analyzed. On the one hand, the dimension of the streamwise component can be set based on previous knowledge of the problem analyzed. In particular, we limit the analysis to the boundary layer, avoiding the leading edge with high curvature, and consider an extension that reaches the fixed transition point (where the turbulent model is switched on). The following intervals are good choices for the upper surface, $0.16 \leq x / c \leq 0.6$, and for the lower surface, $0.2401 \leq x / c \leq 0.6$. The following interval would also be a good and general choice for the normal direction
$0 \leq y / c \leq \pm 0.025(y / c=0$ is the reference value of the upper and lower surfaces of the airfoil, where $x-y$ define a local coordinate system on the airfoil surface). The domain used in the DMD analysis may influence the results obtained; however, the robustness of HODMD ensures that the results do not change, if the parameter $d$ is properly adjusted, as explained next. An initial snapshot matrix is constructed at this step for both upper and lower surfaces, of dimension $\left(n_{x} \times n_{y}\right) 1600 \times 200$ and $1400 \times 200$, respectively, for each velocity component (streamwise and normal velocity).

- Settings for the sequential spatial HODMD analysis. The method will be applied to various small regions of the computational domain (selected in the previous step) and referred to as $I$. These regions are limited by the interval $x_{i} \leq x<x_{i}+\delta x$, with $i=1, \ldots, I$. The accuracy of the results is inversely proportional to the size of this interval. A good estimation to fix this interval size is setting $\delta x$ to be proportional to the number of snapshots $K$ and their relative sampling distance $\Delta \hat{x}$ such that $\delta x=K \cdot \Delta \hat{x}$. After some calibration, based on the accuracy of the obtained results, the number of snapshots is set to $K=20$, and the distance as $\delta x=8 \Delta \hat{x}$ [smaller distances may introduce errors due to the interpolation carried out in the previous step since the parameter $d$, representing the sliding window process in Eq. (6) and responsible for smoothing the results, is proportional to the size of the domain-window and the number of points; smaller dimensions imply smaller values of $d$ and increased noise]. Additionally, it is necessary to consider the number of small subregions $I$ to perform the analysis and where to fix the position $x_{i}$. The analysis is performed in sliding windows $A_{i}$ (as shown in Fig. 4, bottom) where the initial point of each interval is fixed as $x_{i+1}=x_{i}+(i) \cdot L$, where $L$ is a fixed number of snapshots, of the initial snapshot matrix calculated in the previous step. This process may increase the accuracy in the calculations of the $N$-factor while maintaining the number of snapshots $K$ and the parameter $d$ (fixed in Sec. IV). The reason is that the downstream point of the interval $x_{i}+\delta x$ determines the streamwise variations of $N$ and consequently smaller distances between intervals, provide more accurate results. Two different test cases are included below, for $L=20$ (where the maximum number of (a)

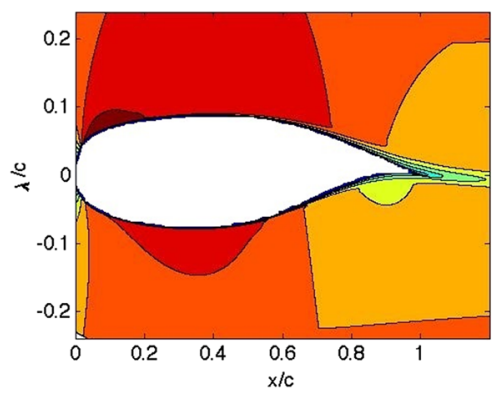

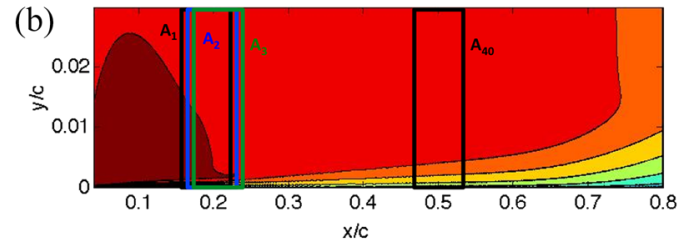

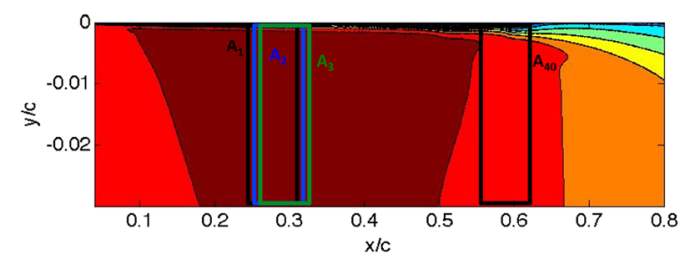

FIG. 4. Contours of streamwise velocity of the two-dimensional NLF0416 airfoil in the interpolated equispaced mesh using $\Delta x=4.002 \cdot 10^{-4}$ and $\Delta y$ $=1.2508 \cdot 10^{-4}$. (a): two dimensional profile defined in the Cartesian coordinates $x-\lambda$. (b): domain used in the HODMD analysis for the upper (top) and lower (bottom) surfaces of the airfoil. The surface of the airfoil is defined for $y / c$ $=0$, where $x$ - $y$ define a local coordinate system on the airfoil surface. 
regions is $I=73$ and $I=63$ for the upper and lower surfaces, respectively) and $L=10$.

- Settings for the HODMD parameters. The calibration process in HODMD is mainly based on the robustness of the results: real physical modes are always retained by the method using different tolerances and values of $d$, while spurious modes are continuously changing. On the one hand, the parameter $d$ should be big enough to clean the noise, related to spurious small fluctuations and noise coming from the data interpolation. However, this parameter should also be small enough to enable the largest wavelength to develop in the $K$ - $d$ snapshots used to form operator (8). If the dynamics are unknown, one can propose an initial guess $K / 10<d<$ $K / 2$, based on previous work. ${ }^{25}$ After some calibration, minimizing the RMS reconstruction error, ${ }^{24}$ it is easily found that $d=8$ is the best choice for $K=20$. It is remarkable that this parameter is proportional to the number of snapshots collected, so similar results can be obtained when using $K^{\prime}=m \cdot K$ snapshots and $d^{\prime}=m \cdot d$ (with $m \in \mathbb{N}^{+}$). On the other hand, the tolerances $\varepsilon_{1}$ and $\varepsilon$ should be smaller than the order of magnitude of the fluctuations. So, in this calibration process, one can use $\varepsilon_{1}=\varepsilon=10^{-8}$ as tolerance, finding that the level of the fluctuations (amplitude of DMD modes) is $\sim 10^{-4}$ to $10^{-3}$, while the order of magnitude for the mean flow is $\sim 1$.

Once the main parameters are set for the analysis, the most unstable spatial mode, related to flow transition, may be identified. The snapshot matrix (2) for the HODMD analysis is conformed using the two velocity components $\left(\boldsymbol{u}^{1}, \boldsymbol{u}^{2}\right)$. Based on the robustness of HODMD, the wavenumber of this mode is easily identified by comparing the results obtained for different tolerances $\varepsilon_{1}$ and $\varepsilon$. Figure 5(a) shows the results obtained in the upper surface for three different test cases using tolerances $\varepsilon_{1}=10^{-8}$ and $\varepsilon=10^{-4} ; \varepsilon_{1}=10^{-6}$ and $\varepsilon=10^{-4}$; and $\varepsilon_{1}=10^{-4}$ and $\varepsilon=10^{-3}$ (similar results, not shown for the sake of brevity, are obtained with other tolerances, e.g., $\varepsilon_{1}=10^{-7}$ and $\varepsilon_{1}=10^{-4}$ for values of $\varepsilon \leq 10^{-3}$ ). This figure shows the wavenumbers and the amplitudes of all the results obtained in $I=40$ regions using $L=20$ (similar results are obtained with $L=10$, not shown). As seen, it is possible to distinguish the mode associated with the base flow, whose amplitude is larger than $10^{-2}$ and the mode related to the fluctuations, whose amplitudes are smaller than $\sim 10^{-3}$. The mode that leads to flow transition is the one related to the fluctuation with the highest amplitude. Since the level of the fluctuations is several orders of magnitude smaller than the base flow, we also find several spurious modes. However, the leading mode, will be always present (robustness in the analysis). It is possible to distinguish 5 modes, referred to as $M 1, M 2, M 3, M 4$, and $M 5$, with amplitudes $\sim 10^{-3}$ that are present in the three cases included. However, there is only one, $M 1$, with dimensional wavenumber $\lambda /(2 \pi)$ $\simeq 44 \mathrm{~m}^{-1}$ that is consistently robust (assuming an error of $2 \%$ in the calculation of the wavenumber, this figure shows that the same mode is found in several regions $A_{i}$ ). The mode $M 5$ is also robust; however, its amplitude is smaller, meaning that $M 5$ is not relevant for our analysis. Finally, the remaining modes $M 2, M 3$, and $M 4$ are not found again in any of the sections $A_{i}$. Figure 5(b) shows the same results obtained using DMD-1, which corresponds to the classic DMD. ${ }^{39}$ As seen, the method is less robust, and it is not possible
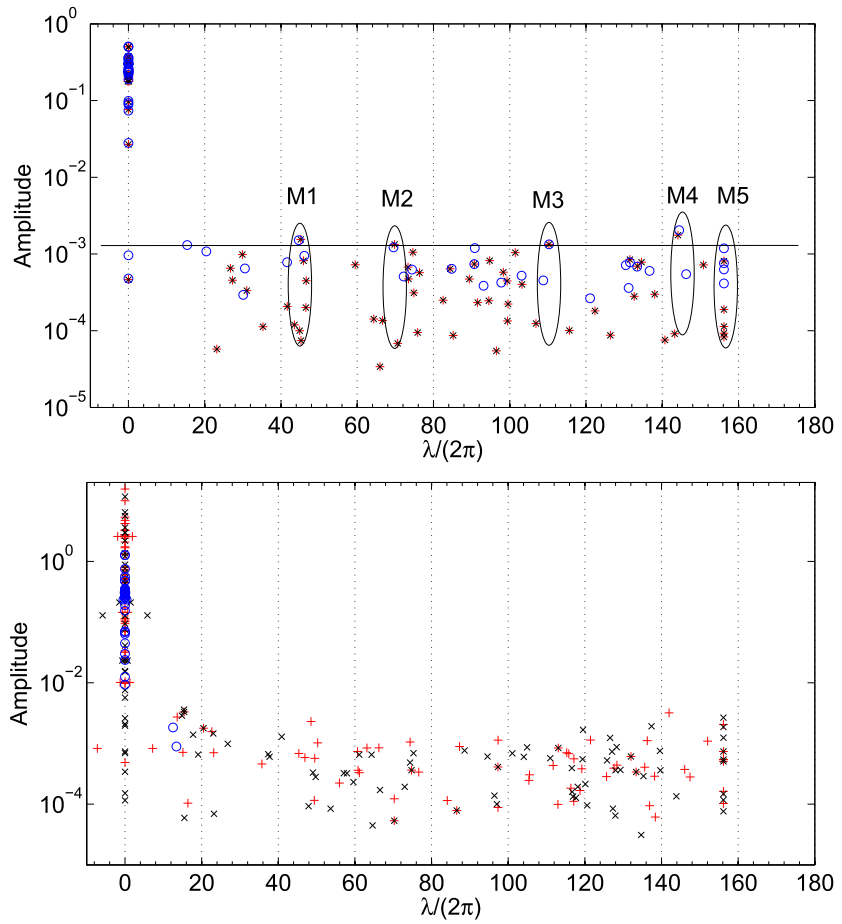

FIG. 5. Amplitude vs wavenumber in the HODMD calculations in the upper surface of the two-dimensional NLF0416 airfoil in $I=40$ regions using $L=20$ : (a) DMD8 and (b) DMD-1. The various symbols represent the results obtained using the following tolerances: blue circles for $\varepsilon_{1}=10^{-4}$ and $\varepsilon=10^{-3}$, black crosses for $\varepsilon_{1}=10^{-6}$ and $\varepsilon=10^{-4}$, and red crosses for $\varepsilon_{1}=10^{-8}$ and $\varepsilon=10^{-4}$.

to distinguish the leading mode $M 1$. A similar analysis has been performed for the lower surface, not shown for brevity, to find that the wavenumber of the leading mode is $\lambda /(2 \pi) \simeq 63 \mathrm{~m}^{-1}$. Finally, let us note that our flat plate estimates of Sec. II C predicted $\lambda /(2 \pi)$ $\simeq 100 \mathrm{~m}^{-1}$, which are not far from the simulated results.

Finally, once the leading mode has been identified, it is necessary to compute the spatial growth of the leading instability, using the computed $N$-factor, as derived in Eq. (17). This value is determined by the variation of the amplitudes as $N \simeq \log \left(a_{l}^{x} / a_{0}\right)$, where $a_{l}^{x}$ is the amplitude of the mode (obtained with HODMD) and $a_{0}$ is the reference amplitude. The level of convergence of the steady flow is considered as reference for the growing amplitudes; in this example, $a_{0}=5 \cdot 10^{-7}$.

\section{RESULTS AND DISCUSSION}

\section{A. Two-dimensional validation: Low-speed laminar NLF0416 airfoil}

In this section, the performance of the method is validated studying the two-dimensional flow transition over a low-speed NLF0416 airfoil, and using the parameter calibrated in Sec. III C.

Figure 6 shows the evolution of the leading mode along the streamwise component using three different parameters in the upper surface (a) and lower surface (b). The curves are obtained via leastsquare fitting to the extracted points. As seen, the mode grows to 
(a)

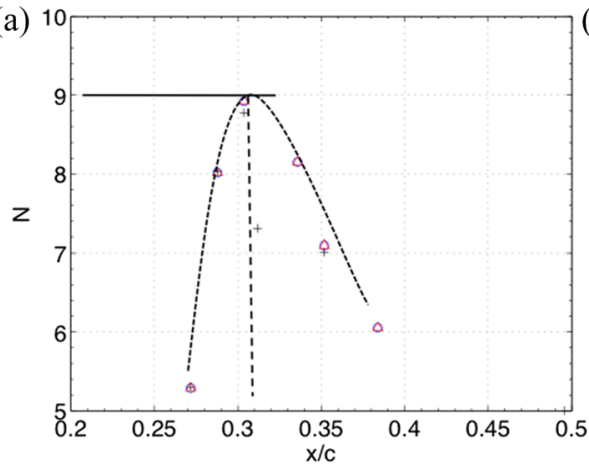

(b)

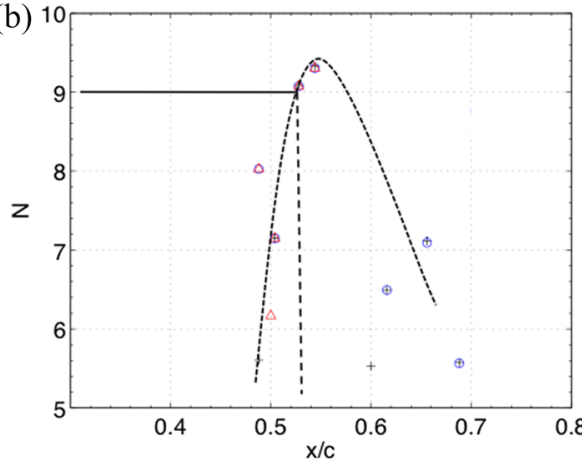

FIG. 6. Evolution of the $N$-factor of the leading mode as a function of the streamwise component $x / c$ in the twodimensional NLF0416 airfoil. The $\mathrm{N}$ factor is calculated as $N=\log \left(a_{1}^{x} / a_{0}\right)$ (a) upper surface and (b) lower surface. The various symbols represent the results obtained using the following parameters with DMD-8: black crosses for $\varepsilon_{1}=10^{-6}, \varepsilon=10^{-4}$, and $L=20$; blue circles for $\varepsilon_{1}=10^{-4}, \varepsilon=10^{-4}$, and $L=20$; and red triangles for $\varepsilon_{1}=10^{-4}$, $\varepsilon=10^{-4}$, and $L=10$. then decay. Due to noise artefacts, it is possible to find some spurious (or not accurately calculated) points; however, the three cases match the results in the growing region of the curve, showing the robustness of the analysis. Therefore, the spurious results are easily identified. The transition point, when selecting a critical $N$-factor $N_{\text {crit }}=9$, is found at $x / c \sim 0.31$ in the upper surface and $x / c \sim 0.52$ in the lower surface. These results are in good agreement with the linear stability theory and the experimental results, ${ }^{28,48}$ as summarized in Table II.

Having validated the method with a suitable set of parameters, we proceed to calculate transition points over swept wings.

\section{B. Three-dimensional wing with sweep angle based on the laminar NACA64 2 A015 airfoil}

The origin of cross-flow transition is a secondary instability developing along the streamwise direction. To study this secondary instability, it is necessary to first identify the modes related to the primary instability, and leading to spanwise oscillations. Once these modes are identified, the same analysis performed in Sec. IV A will be applied to the highest amplitude spanwise-mode [which is twodimensional, see Eq. (10)]. Therefore, as explained in Sec III B, this analysis will be carried out in two different steps.

Three-dimensional numerical simulations have been performed to study the cross flow transition of a wing composed of NACA64 2 A015 airfoils. The two dimensional profile has been extruded in the streamwise direction, with a spanwise mesh spacing of $\Delta z=0.0435 \mathrm{~m}$. The boundary conditions set in the previous two dimensional analysis are maintained, and periodic boundary conditions are applied in both sides of the spanwise direction. The solution has been converged to steady state with the residuals set to $5 \cdot 10^{-7}$ using nonlinear Navier-Stokes equations, so the solution will take into account nonlinear interactions.

TABLE II. Estimation of the transition point in the upper and lower surfaces of the two-dimensional NLF0416 airfoil using DMD-8, linear stability theory (LST) and experiments (EXPT.). $\mathrm{N}$-factor for transition is fixed to $\mathrm{N}=9$.

\begin{tabular}{lcc}
\hline \hline Method & x/c (up) & $\mathrm{x} / \mathrm{c}$ (low) \\
\hline HODMD & 0.31 & 0.52 \\
LST & 0.33 & 0.568 \\
EXPT. & $0.35-0.40$ & $0.5-0.55$ \\
\hline \hline
\end{tabular}

The cross-flow angle is set in the mesh with respect to the spanwise direction as $\Lambda=10^{\circ}$ and $40^{\circ}$, as illustrated in Fig. 3. The mesh is structured, but not equispaced in the streamwise and normal directions. So, as explained in Sec. IV A, the three-dimensional computational domain has been interpolated to a structured mesh, using the same parameters as before $\left(\Delta x=4.002 \cdot 10^{-4} \mathrm{~m}\right.$ and $\Delta y$ $=1.2508 \cdot 10^{-4} \mathrm{~m}$, and the spanwise structure is maintained captured using $\Delta z=0.0435 \mathrm{~m}$ ). The HODMD can now be applied to the structured mesh along the spanwise direction. The snapshot matrix (2) is conformed using the three velocity components $\left(\boldsymbol{u}^{1}, \boldsymbol{u}^{2}, \boldsymbol{u}^{3}\right)$. Figure 7 shows the amplitudes as a function of the wavenumbers obtained in the HODMD analysis using 24 flow fields on z-planes. The tolerances set for this analysis is $\varepsilon_{1}=\varepsilon=10^{-8}$. As seen in this figure, the flow is periodic in the spanwise direction, as expected, which is confirmed when analyzing the spanwise growth rates which are all close to zero. HODMD identifies the mean flow (whose wavenumber is 0 ), a single mode with wavenumber $\beta /(2 \pi)=1$ and ten harmonics (and complex conjugates). Due to the simplicity of this analysis, and the low number of snapshots used, accurate results are obtained for values of the HODMD parameter $1 \leq d \leq 5$.

In the second step, the highest amplitude mode [with dimensional spanwise wavenumber $\beta /(2 \pi)=1 \mathrm{~m}$ ] is selected, and a HODMD analysis is performed upon this mode and along the streamwise direction. Note that we have checked the growth of secondary instabilities in all spanwise wavelengths to find that the dominant secondary instabilities live in the longest spanwise wavelength. The secondary mode is now two-dimensional, and consequently the analysis is similar to the previous two-dimensional analysis for the NLF0416 airfoil. The same methodology and setting parameters are used for this analysis (upper surface). However, the reference value for the amplitudes is slightly smaller than before, $a_{0}=10^{-6}$. The reason is that, due to numerical errors (interpolation and HODMD spanwise analysis), some accuracy might be lost in the calculations of the first analysis (the reference value for the numerical simulations was $5 \cdot 10^{-7}$ ). The reference value $a_{0}$ can be checked by performing the HODMD analysis in the two-dimensional mode (using the complete section) along the streamwise direction using the tolerances $\varepsilon_{1}=\varepsilon=10^{-8}$ (machine precision). The order of magnitude of the lowest amplitude mode would be set as a reference value for the analysis.

Figure 8 (a) shows the evolution of the leading mode for a sweep angle of $\Lambda=10^{\circ}$, along the streamwise component using $L=20$ and $\varepsilon_{1}=\varepsilon=10^{-4}$. As expected, the mode grows up to the transition point, 


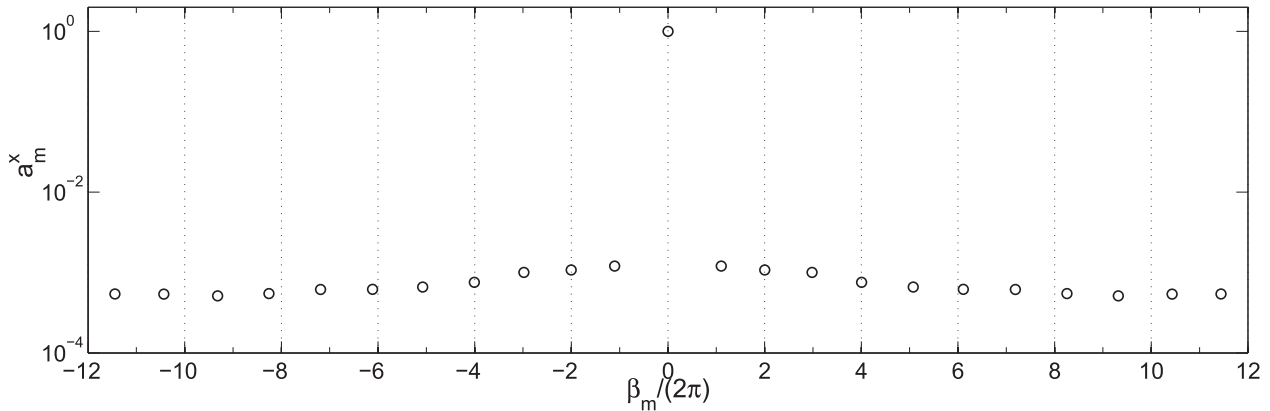

(a)

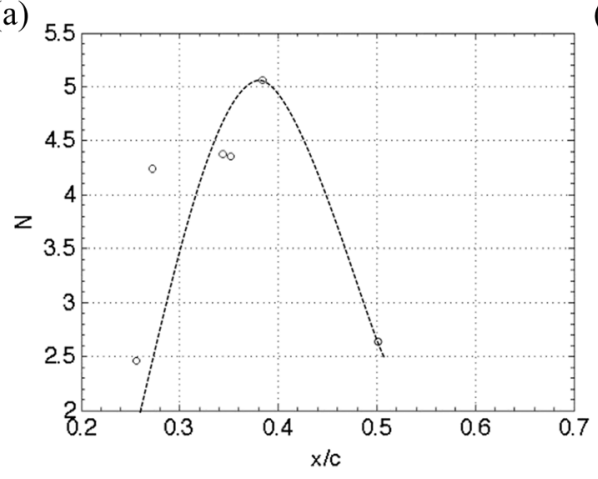

(b)

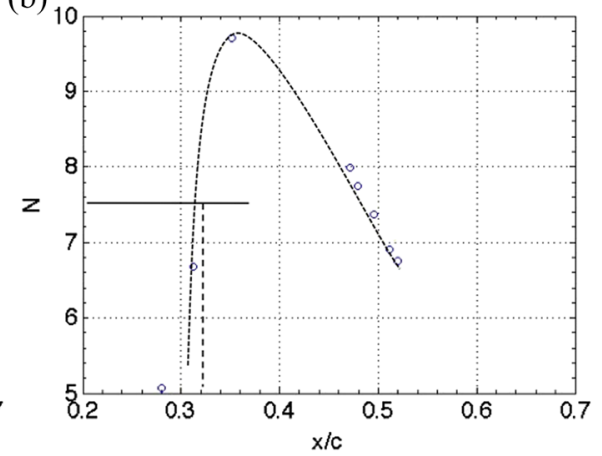

FIG. 7. Amplitudes vs wavenumbers obtained with DMD-d $(1 \leq d \leq 5)$ with tolerances $\varepsilon_{1}=\varepsilon=10^{-8}$ applied in the spanwise direction of a threedimensional airfoil with cross-flow angle $\Lambda=40^{\circ}$. to then decay. For this low sweep angle, there is no transition when fixing the critical $N$-factor to $N_{c r i t}=7.5$ and we only find transition at $x / c \simeq 0.38$ for a lower value of the critical $N$-factor $N_{\text {crit }}=5$. When the analysis is performed for a higher sweep angle $\Lambda=40^{\circ}$, Fig. 8(b), then the transition location for $N_{c r i t}=7.5$ is $x / c \simeq 0.32$ and almost identical to when increasing the critical critical $N$-factor to $N_{c r i t}=9.5$ leading to $x / c \simeq 0.34$. These values agree well with the experiments reported in Ref. 6, which reported $x / c \simeq 0.3$.

For completeness, we compute the $N$-factor also for $\Lambda=40^{\circ}$ and $\operatorname{Re}=6.35 \cdot 10^{6}$ and show the resulting curve in Fig. 9. In this case, we find that for $N_{\text {crit }}=7.5$ transition occurs at $x / c \simeq 0.39$.

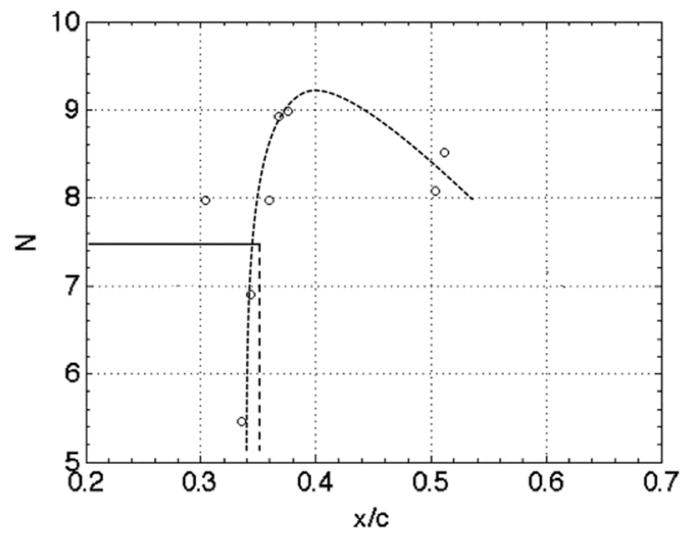

FIG. 9. $\mathrm{N}$-factor vs streamwise direction $x / \mathrm{c}$ for swept wing at $A O A=-1^{\circ}$ and $\operatorname{Re}=6.35 \cdot 10^{6}$ and sweep angle $\Lambda=40^{\circ}$. DMD-8 with tolerances $\varepsilon_{1}=10^{-6}$, $\varepsilon=10^{-4}$, and $L=20$.
At these conditions, experimental measurements and the LST modified for cross-flow ${ }^{51}$ (with $N_{\text {crit }}=7.5$ ) predicted transition locations at $x / c \simeq 0.44$ and $x / c \simeq 0.4$, respectively.

In it interesting to note that our results suggest an alternative to using a fixed critical $N$-factor to detect the transition point.

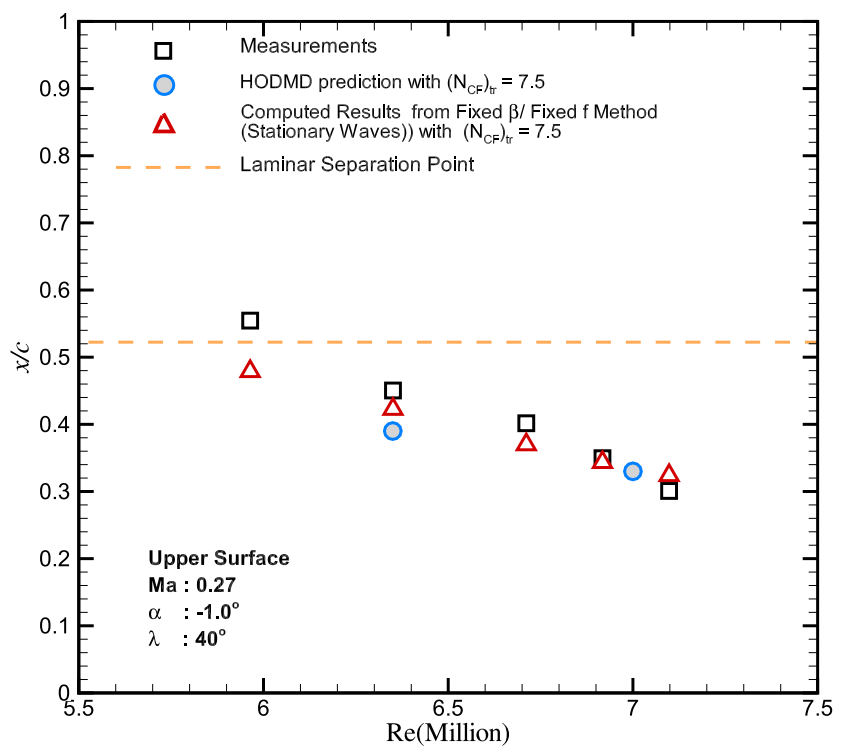

FIG. 10. Transition point vs Reynolds number for sweep wing with angle $\Lambda=40^{\circ}$ at $A \circ A=-1^{\circ}$. The figure includes experimental measurements, ${ }^{6} \mathrm{HODMD}$ results, and results from an LST modified method to account for cross-flow. 
Transition location could be approximated by the maximum peak of the $N$-factor curve (or when the first derivative is zero: $d N / d x=$ $0)$. In fact, when using the curve maxima, for all tested cases, we obtain better matches to experiments than when using the critical $N$-factors, this last idea needs further study and may be linked to more general criterion to determine the transition threshold, such as the "Variable N-Factor technique."

Finally, we summarize the cross-flow results in Fig. 10, where our HODMD results are compared against experimental data ${ }^{6}$ and LST modified for cross-flow ${ }^{51}$ (with $N_{\text {crit }}=7.5$ ) for various Reynolds numbers at a sweep angle of $\Lambda=40^{\circ}$. Our results agree well with measurements demonstrating the potential of this new approach to predict cross-flow instabilities.

\section{CONCLUSIONS}

We have presented a new method to analyze cross-flow instabilities based on a spatial HODMD analysis and steady base flow simulations. We have shown that the method can distinguish between TS and cross-flow type transition scenarios and provide $N$-factors that can be coupled with semiempirical criteria to determine the transition point.

The method does not require modeling of the perturbation but instead extracts the instability information from the steady flow field. The method is automatic and robust and can be applied to a section of the computational domain without the necessity of specifying streamlines of initial amplitudes for perturbations, although some initial calibration is required to adjust the parameters of the method. Additionally, the method can potentially account for mode interactions, surface imperfections, ${ }^{42}$ and polychromatic transitional scenarios $^{43}$ that are difficult to predict with existing methods.

\section{ACKNOWLEDGMENTS}

This research was partially supported by the Universidad Politécnica de Madrid I +D+I Program for International Collaboration.

\section{REFERENCES}

${ }^{1}$ D. Arnal and G. Casalis, "Laminar-turbulent transition prediction in threedimensional flows," Prog. Aerosp. Sci. 36, 173-191 (2000).

${ }^{2} \mathrm{H}$. Bippes, "Basic experiments on transition in three-dimensional boundary layers dominated by crossflow instability," Prog. Aerosp. Sci. 35, 363-412 (1999).

${ }^{3}$ V. I. Borodulin, A. V. Ivanov, and Y. S. Kachanova, "Swept-wing boundary-layer transition at various external perturbations: Scenarios, criteria, and problems of prediction," Phys. Fluids 29(9), 094101 (2017).

${ }^{4}$ R. Bose and P. A. Durbin, "Transition to turbulence by interaction of free-stream and discrete mode perturbations," Phys. Fluids 28(11), 114105 (2016).

${ }^{5}$ L. N. Cattafesta III and M. Sheplak, "Actuators for active flow control," Annu. Rev. Fluid Mech. 43(1), 247-272 (2011).

${ }^{6}$ F. W. Boltz, G. C. Kenyon, and C. Q. Allen, "Boundary-layer stability characteristics of an untapered wing at low speeds," Report No. NASA-TN-D-338, NASA Ames Research Center, Moffett Field, California, 1960.

${ }^{7}$ L. De Lathawer, B. De Moor, and J. Vandewalle, "A multilinear singular value decomposition,” SIAM J. Matrix Anal. Appl. 21, 1253-1278 (2000).

${ }^{8} \mathrm{U}$. Ehrenstein and F. Gallaire, "On two-dimensional temporal modes in spatially evolving open flows: The flat-plate boundary layer," J. Fluid Mech. 536, 209-218 (2005).

${ }^{9}$ A. Fedorov, "Transition and stability of high-speed boundary layers," Annu. Rev. Fluid Mech. 43(1), 79-95 (2011).
${ }^{10}$ E. Ferrer, J. DeVicente, and E. Valero, "Low cost three-dimensional global instability analysis and flow sensitivity based on dynamic mode decomposition and high-order numerical tools," Int. J. Numer. Methods Fluids 76(3), 169-184 (2014).

${ }^{11}$ L. M. Gonzalez, J. M. Gimenez, and E. Ferrer, "Instability onset for a submerged cylinder," Phys. Fluids 31(1), 014106 (2019).

${ }^{12}$ L. M. Gonzalez, E. Ferrer, and H. R. Diaz-Ojeda, "Onset of three dimensional flow instabilities in lid-driven circular cavities,” Phys. Fluids 29, 064102 (2017).

${ }^{13}$ Z. H. Han, J. Chen, K. S. Zhang, Z. M. Xu, Z. Zhu, and W. P. Song, "Aerodynamic shape optimization of natural-laminar-flow wing using surrogate-based approach," AIAA J. 56(7), 2579-2593 (2018).

${ }^{14}$ Z. H. Han, F. He, W. P. Song, and Z. D. Qiao, "A preconditioned multigrid method for efficient simulation of three dimensional compressible and incompressible flows," Chin. J. Aeronaut. 20(4), 289-296 (2007).

${ }^{15}$ Z. H. Han, C. Z. Xu, L. Zhang, Y. Zhang, K. S. Zhang, and W. P. Song, "Efficient aerodynamic shape optimization using variable-fidelity surrogate models and multilevel computational grids," Chin. J. Aeronaut. (in press).

${ }^{16}$ T. Herbert and N. Lin, "Studies on boundary-layer receptivity with parabolized stability equations," AIAA Paper No. 93-3053, 1993.

${ }^{17}$ T. Herbert, "Parabolized stability equations," Annu. Rev. Fluid. Mech. 29, 245283 (1997).

${ }^{18} \mathrm{~W}$. Koch, "On a degeneracy of temporal secondary instability modes in Blasius boundary-layer flow," J. Fluid Mech. 243, 319-351 (1992).

${ }^{19}$ Y. Kohama, T. Onodera, and Y. Egami, "A high-frequency, secondary instability of crossflow vortices that leads to transition," in Proceedings of Royal Aerospace Society Conference Boundary-Layer Transition Control Conference (Peterhouse College, Cambridge, UK, 1991), pp. 4.1-4.13.

${ }^{20}$ T. G. Kolda and B. W. Bader, "Tensor decompositions and applications," SIAM Rev. 51, 455-500 (2009).

${ }^{21}$ S. Le Clainche and E. Ferrer, "Reduced order model to predict transient flows around straight bladed vertical axis wind turbines," Energies 11(3), 566 (2018).

${ }^{22}$ S. Le Clainche, J. M. Pérez, and J. M. Vega, "Spatio-temporal flow structures in the three-dimensional wake of a circular cylinder," Fluid Dyn. Res. 50, 051406 (2018).

${ }^{23}$ S. Le Clainche, D. Rodríguez, V. Theofilis, and J. Soria, "Flow around a hemisphere-cylinder at high angle of attack and low Reynolds number. Part II: POD and DMD applied to reduced domains," Aerosp. Sci. Technol. 44, 88-100 (2015).

${ }^{24}$ S. Le Clainche, F. Sastre, J. M. Vega, and A. Velazquez, "Higher order dynamic mode decomposition applied to study flow structures in noisy PIV experimental data," AIAA Paper 2017-3304, 2017.

${ }^{25}$ S. Le Clainche and J. M. Vega, "Higher order dynamic mode decomposition," SIAM J. Appl. Dyn. Syst. 16(2), 882-925 (2017).

${ }^{26} \mathrm{~S}$. Le Clainche and J. M. Vega, "Higher order dynamic mode decomposition to identify and extrapolate flow patterns," Phys. Fluids 29(8), 084102 (2017).

${ }^{27}$ S. Le Clainche, J. M. Vega, and J. Soria, "Higher order dynamic mode decomposition of noisy experimental data: The flow structure of a zero-net-mass-flux jet," Exp. Therm. Fluid Sci. 88, 336-353 (2017).

${ }^{28}$ S. Le Clainche, M. M. Wu, Z. H. Han, and E. Ferrer, "An alternative method to calculate cross-flow instabilities," AIAA Paper 2018-3700, 2018.

${ }^{29}$ M. R. Malik and F. Li, "Secondary instability of Gortler and crossflow vortices," in Proceedings of International Symposium Aerospace \& Fluid Science (Tohoku University, Sendai, Japan, 1993), pp. 460-477.

${ }^{30}$ M. R. Malik, F. Li, M. M. Choudhari, and C. L. Chang, "Secondary instability of crossflow vortices and swept-wing boundary layer transition," J. Fluid Mech. 399, 85-115 (1999).

${ }^{31}$ D. I. A. Poll, "Some observations of the transition process on the windward face of a long yawed cylinder," J. Fluid Mech. 150, 329-356 (1985).

${ }^{32}$ H. Raposo, S. Mughal, and R. Ashworth, "Acoustic receptivity and transition modeling of Tollmien-Schlichting disturbances induced by distributed surface roughness," Phys. Fluids 30(4), 044105 (2018).

${ }^{33}$ H. L. Reed, E. Perez, J. J. Kuehl, T. Kocian, and N. Oliviero, "Verification and validation issues in hypersonic stability and transition prediction," J. Spacecr. Rockets 52(1), 29-37 (2015). 
${ }^{34}$ W. S. Saric, H. L. Reed, and E. B. White, "Stability and transition of threedimensional boundary layers,” Annu. Rev. Fluid. Mech. 35, 413-440 (2003).

${ }^{35}$ W. S. Saric, A. L. Carpenter, and H. L. Reed, "Passive control of transition in three-dimensional boundary layers, with emphasis on discrete roughness elements,” Philos. Trans. R. Soc., A 369, 1352-1364 (2011).

${ }^{36} \mathrm{H}$. Schlichting and K. Gersten, "Fundamentals of turbulent flows," in BoundaryLayer Theory, 8th revised and enlarged edition (Springer, Berlin, 2000), pp. 499516.

${ }^{37}$ L. Sirovich, "Turbulence and the dynamics of coherent structures. Parts I-III," Q. Appl. Math. 45(3), 561-571 (1987).

${ }^{38} \mathrm{H}$. Schlichting, "Zur entstehung der turbulenz bei der plattenstromung," in Nachrichten von der Gesellschaft der Wissenschaften zu Göttingen, MathematischPhysikalische Klasse (Niedersäechsische Staats- und Universitäetsbibliothek, Gottingen, 1933), pp. 181-208.

${ }^{39} \mathrm{P}$. J. Schmid, "Dynamic mode decomposition of numerical and experimental data," J. Fluid Mech. 656, 5-28 (2010).

${ }^{40}$ L. Schrader, S. Amin, and L. Brandt, "Transition to turbulence in the boundary layer over a smooth and rough swept plate exposed to free-stream turbulence," J. Fluid Mech. 646, 297-325 (2010).

${ }^{41}$ L. Schrader, L. Brandt, and D. Henningson, "Receptivity mechanisms in threedimensional boundary-layer flows," J. Fluid Mech. 618, 209-241 (2009).

${ }^{42}$ P. K. Sharma and T. K. Sengupta, "Effect of frequency and wavenumber on the three-dimensional routes of transition by wall excitation," Phys. Fluids 31(6), 064107 (2019).
${ }^{43}$ P. Sundaram, T. K. Sengupta, and S. Sengupta, "Is Tollmien-Schlichting wave necessary for transition of zero pressure gradient boundary layer flow," Phys. Fluids 31(3), 031701 (2019).

${ }^{44}$ D. Tempelmann, L. U. Schrader, A. Hanifi, L. Brandt, and D. S. Henningson, "Swept wing boundary-layer receptivity to localised surface roughness," J. Fluid Mech. 711, 516-544 (2012).

${ }^{45} \mathrm{~W}$. Tollmien, "Uber die entstehung der turbulenz," in Nachrichten von der Gesellschaft der Wissenschaften zu Göttingen, Mathematisch-Physikalische Klasse (Niedersäechsische Staats- und Universitäetsbibliothek, Gottingen, 1929), pp. 21-44.

${ }^{46}$ L. R. Tucker, "Some mathematical notes on three-mode factor analysis," Psikometrica 31, 279-311 (1996).

${ }^{47}$ M. Wang, T. Herbert, and G. K. Stuckert, "PSE analysis of receptivity and stability in swept wing flows," AIAA Paper No. 94-0180, 1994.

${ }^{48}$ M. Wu, Z. Han, H. Nie, W. Song, S. Le Clainche, and E. Ferrer, "A transition prediction method for flows over airfoils based on dynamic mode decomposition," Chin. J. Aeronaut. (in press).

${ }^{49}$ D. Xiao and G. Papadakis, "Nonlinear optimal control of bypass transition in a boundary layer flow method," Phys. Fluids 29(5), 054103 (2017).

${ }^{50}$ F. Xie, W. P. Song, and Z. H. Han, "Numerical study of high-resolution scheme based on preconditioning method," J. Aircraft 46(2), 520-525 (2009).

${ }^{51}$ K. S. Zhang, Z. H. Han, Z. J. Gao, and Y. Wang, "Constraint aggregation for large number of constraints in wing surrogate-based optimization," Struct. Multidiscip. Optim. 59(2), 421-438 (2019). 